\title{
OPEN Subthreshold laser treatment for reticular pseudodrusen secondary to age-related macular degeneration
}

\author{
Giuseppe Querques ${ }^{1,2,3,4 \rrbracket}$, Riccardo Sacconi ${ }^{1,2,4}$, Francesco Gelormini ${ }^{1,2}$, Enrico Borrelli, ${ }^{1,2}$, \\ Francesco Prascina ${ }^{2}$, Ilaria Zucchiatti ${ }^{2}$, Lea Querques ${ }^{2}$ \& Francesco Bandello ${ }^{1,2}$
}

There is a lack of treatment aimed at the regression of reticular pseudodrusen (RPD) secondary to agerelated macular degeneration (AMD). The aim of this prospective, pilot study is to evaluate the safety and short-term efficacy of subthreshold laser treatment (SLT) in patients affected by RPD secondary to dry AMD (dAMD). Twenty eyes of 20 patients (mean age $78.4 \pm 6.8$ years) with RPD secondary to dAMD were prospectively enrolled. All patients were treated in an extrafoveal area of $1.27 \mathrm{~mm}^{2}$ using end-point management yellow subthreshold laser and followed for 3 months. Best-corrected visual acuity was $0.140 \pm 0.09$ LogMAR at the baseline and no changes were observed during the follow-up $(p=0.232)$. No significant worsening was disclosed before and after the treatment analyzing the macular sensitivity of the treated area $(p=0.152)$. No topical and/or systemic side effects were disclosed during the 3-month follow-up. The distribution among the RPD stages changed after the treatment $(p<0.001)$. In detail, in the treated area, we observed a significant increase in the number of Stage 1 RPD during the follow-up $(p=0.002)$, associated with a significant decrease of Stage 3 $\operatorname{RPD}(p=0.020)$. Outer nuclear layer (ONL) thickness analysis showed a significant increase after the treatment associated with RPD regression $(p=0.001)$. End-point management SLT appears a safe treatment for RPD secondary to dAMD, showing short-term safety outcomes. Our results suggest that SLT could be effective in inducing a RPD regression in terms of RPD stage and ONL thickening.

Age-related macular degeneration (AMD) is a progressive chronic disease and the first cause of vision impairment of elderly people in western countries ${ }^{1,2}$. AMD could be classified in two different forms based on the presence of macular neovascularization (MNV): neovascular AMD (nAMD), and non-neovascular or dry AMD (dAMD).

In the last decades, the introduction in the clinical practice of anti-vascular endothelial growth factor (VEGF) injections has changed the natural history of the nAMD, dramatically reducing the visual loss due to the $\mathrm{MNV}^{3}$. Conversely, no specific treatment is available to avoid the progression of dAMD into its advanced stage, namely geographic atrophy $(\mathrm{GA})^{4,5}$. Dry AMD is characterized by the progressive loss of the photoreceptors, retinal pigment epithelium (RPE) cells, and choriocapillaris in the affected areas ${ }^{6-9}$. As reported by the Age-Related Eye Disease Study (AREDS) and AREDS2 study, dietary supplementation and general lifestyle modification could reduce the risk of AMD progression in high-risk patients affected by dAMD ${ }^{10-12}$. However, no specific therapies that slow the progression from earlier and asymptomatic stages of the disease into the late stage of dAMD are available. For these reasons, several groups have focused their research on this field, trying to propose new strategies for the treatment of this diffuse and devastating disease.

Reticular pseudodrusen (RPD) are one of the findings characterizing the early and intermediate stages of dAMD, together with drusen and RPE changes ${ }^{13}$. The presence of RPD is associated with a worse visual and anatomical function already from the early stages of the disease ${ }^{14-17}$. Furthermore, several studies have highlighted the role of RPD to accelerate the progression to both forms of late AMD ${ }^{14,18,19}$. For these reasons, a treatment aimed at the regression of RPD could be mandatory in order to prevent the progression to late AMD.

\footnotetext{
${ }^{1}$ School of Medicine, Vita-Salute San Raffaele University, Milan, Italy. ${ }^{2}$ Division of Head and Neck, Ophthalmology Unit, IRCCS San Raffaele Scientific Institute, Milan, Italy. ${ }^{3}$ Department of Ophthalmology, University Vita-Salute, IRCCS Ospedale San Raffaele, Via Olgettina 60, 20132 Milan, Italy. ${ }^{4}$ These authors contributed equally: Giuseppe Querques and Riccardo Sacconi. ${ }^{\varpi}$ email: querques.giuseppe@hsr.it
} 


\begin{tabular}{|l|l|l|l|l|}
\hline Visit & Screening & Treatment (baseline) & Follow-up & Follow-up \\
\hline Point of time & Between - 14 days and day 0 & day 0 & Month 1 & Month 3 \\
\hline Assessment & & & & \\
\hline Inclusion/exclusion criteria & $\mathrm{X}$ & & & \\
\hline Informed consent & $\mathrm{X}$ & & & \\
\hline Demographic data & $\mathrm{X}$ & & & \\
\hline Medical history & $\mathrm{X}$ & & & \\
\hline Concurrent medications & $\mathrm{X}$ & & & \\
\hline Ophthalmic history & $\mathrm{X}$ & $\mathrm{X}$ & $\mathrm{X}$ & \\
\hline BCVA at 4 m prior to dilation & $\mathrm{X}$ & $\mathrm{X}$ & $\mathrm{X}$ & $\mathrm{X}$ \\
\hline Slit lamp examination & $\mathrm{X}$ & $\mathrm{X}$ & $\mathrm{X}$ & $\mathrm{X}$ \\
\hline Fundus examination & $\mathrm{X}$ & $\mathrm{X}$ & $\mathrm{X}$ & $\mathrm{X}$ \\
\hline IOP measurement & $\mathrm{X}$ & $\mathrm{X}$ & $\mathrm{X}$ & $\mathrm{X}$ \\
\hline Macular sensitivity by microperimetry & $\mathrm{X}$ & $\mathrm{X}$ & $\mathrm{X}$ & $\mathrm{X}$ \\
\hline FAF by BAF & $\mathrm{X}$ & $\mathrm{X}$ & $\mathrm{X}$ & $\mathrm{X}$ \\
\hline Structural SD-OCT & $\mathrm{X}$ & $\mathrm{X}$ & & \\
\hline Subthreshold laser treatment & & $\mathrm{X}$ & $\mathrm{X}$ & $\mathrm{X}$ \\
\hline Adverse events assessment & & & \\
\hline
\end{tabular}

Table 1. Timing of study assessment. BCVA Best-corrected visual acuity, IOP intra-ocular pressure, FAF fundus autofluorescence, $B A F$ blue autofluorescence, $S D$-OCT spectral-domain optical coherence tomography.

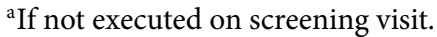

Subthreshold laser is a safe and effective treatment used in the clinical practice in several retinal disorders ${ }^{20-22}$. Although the exact mechanism of action of subthreshold lasers is not completely understood, it has been suggested that it works by targeting, preserving, and "normalizing" the function of the RPE ${ }^{23}$. Since the dysfunction of the RPE has been suggested as the main driving factor in the pathogenesis of RPD, the subthreshold laser could play a crucial role in the treatment of RPD. However, to date, no prospective studies were designed in order to evaluate the safety and efficacy of this treatment in patients affected by RPD. The aim of the current pilot clinical trial is to evaluate the safety and short-term efficacy of the subthreshold laser treatment (SLT) in patients affected by RPD secondary to dAMD.

\section{Methods}

The PASCAL clinical trial is a single-center, non-randomized, pilot study including adults admitted to the Department of Ophthalmology of University Vita-Salute San Raffaele in Milan, Italy, who are suffering from RPD secondary to dAMD.

The trial was conducted in accordance with the Declaration of Helsinki and the study protocol was approved by the Ethics Committee of San Raffaele Hospital (approved on 05/05/2016). The trial was registered on ClinicalTrials.gov (ID NCT02800356, registered on 15/06/2016). All study participants provided written informed consent. The study was conducted in the Medical Retina \& Imaging Unit of the Department of Ophthalmology of University Vita-Salute, IRCCS Hospital San Raffaele in Milan, Italy, between June 2016 and September 2019.

The complete list of the inclusion and exclusion criteria is presented in Supplement 1. Briefly, we included patients aged more than 50 years old, with a diagnosis of dAMD and the presence of RPD. We excluded patients with evidence of GA or MNV in the included eye, any prior treatment for AMD in the included eye (aside from antioxidants), and opacities of the ocular media that not permit high-quality imaging examinations.

In cases where both eyes were eligible, the eye with the worse BCVA at baseline was selected as the study eye. If both eyes have the same BCVA, it was recommended to select the right eye as the study eye.

Subjects had the right to withdraw from the study at any time, for any reason, without jeopardizing their medical care.

Study protocol. The study protocol is summarized in Table 1. After obtaining informed consent and after the screening visit, at the Baseline (Day 0) all patients were evaluated with a complete ophthalmic examination, including Best Corrected Visual Acuity (BCVA) using Snellen charts, slit lamp examination, fundus examination (by indirect ophthalmoscopy) and intraocular pressure measurement (IOP). As imaging protocol, all patients were evaluated using Spectral Domain Optical Coherence Tomography (SD-OCT), fundus autofluorescence (FAF) in the area later treated with laser, microperimetry in a customized area later treated with laser. An extrafoveal area of $1.27 \mathrm{~mm}^{2}\left(1 / 2\right.$ of a disk area, disk area $\left.=2.54 \mathrm{~mm}^{2}\right)$ was treated using yellow subthreshold laser (Pascal Synthesis 577 system, Topcon Corporation, Tokyo, Japan). SD-OCT and FAF images were performed using Spectralis HRA + OCT (Heidelberg Engineering, Heidelberg, Germany), whereas microperimetry was performed using MP-1 (Nidek Technologies, Padova, Italy).

All patients were evaluated at 1-month and 3-month follow-up, with a complete ophthalmic examination, including SD-OCT, FAF, and microperimetry. 


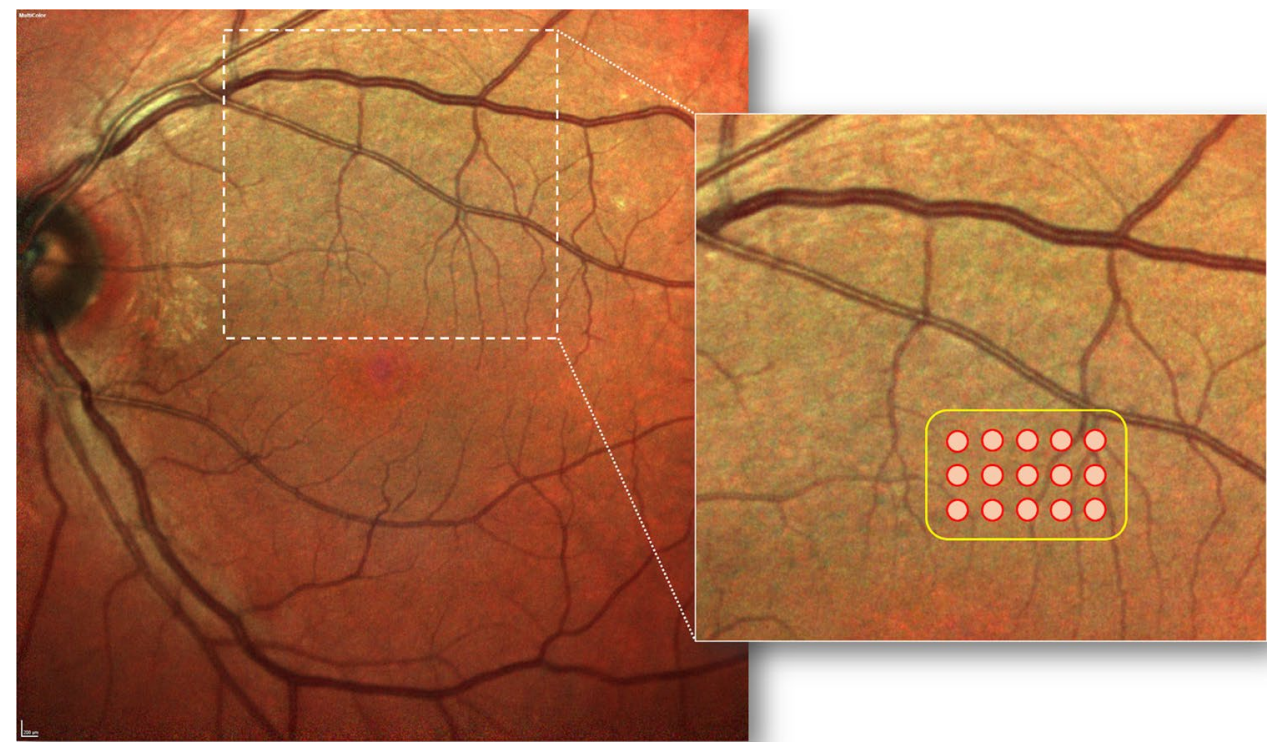

Figure 1. A representative case of an included patient affected by reticular pseudodrusen in the left eye. An extrafoveal area of $1.27 \mathrm{~mm}^{2}\left(1 / 2\right.$ of a disk area, disk area $\left.=2.54 \mathrm{~mm}^{2}\right)$ was selected and treated using yellow subthreshold laser (Pascal Synthesis 577 system, Topcon Corporation, Tokyo, Japan). The treatment was performed using the Endpoint Management ( $30 \%$ of the power of the barely visible burn) with a pattern of $5 \times 3$ spots (area of $1.27 \mathrm{~mm}^{2}$ ).

Subthreshold laser treatment. All the treatments were performed by an expert senior author (GQ). The treatment was performed using the Pascal Synthesis 577 system. During the treatment, the investigator identified the threshold layer within the vascular arcades but outside the central fovea. In detail, the threshold level output power was set to obtain barely visible burn at approximately $200-250 \mathrm{~mW}$ using the titration mode. After that, the investigator identified an area inside the vascular arcades affected by RPD. The irradiation was conducted on this area after switching over to Endpoint Management $(\sim 30 \%$ of the power of the barely visible burn) with a pattern of $5 \times 3$ spots (area of $1.27 \mathrm{~mm}^{2}$ ). Figure 1 shows a representative fundus schema and laser area of the treatment.

Fundus autofluorescence and spectral domain optical coherence tomography. FAF images were used to detect the possible appearance of GA (defined as a hypo-autofluorescence area) in the treated area during the follow-up. SD-OCT images were used to analyze the outer retinal morphology in the treated area, including evaluation of the stages of RPD at the baseline and during the follow-up as previously reported ${ }^{24}$, and the thickness of the outer nuclear layer (ONL) at the baseline and during the follow-up. Briefly, stage 1 RPD are characterized by diffuse deposition of hyperreflective material between the RPE and the inner/outer segments (IS/OS) boundary; stage 2 is characterized by accumulated material that alters the IS/OS boundary; stage 3 is characterized by thicker and conical appearance of deposited material passing through the IS/OS boundary; stage 4 is characterized by fading of the material due to the reabsorption and migration within the inner retinal layers ${ }^{24}$. The ONL thickness was manually measured in the correspondence of each RPD in the treated area; the mean value was considered for the statistical analysis. All measurements were performed by two independent and experienced readers (FG and EB). The grading of the RPD stage was performed by the same two expert readers. In those cases in which the two graders did not agree on a single consensus result, the final decision was performed by a senior author (GQ).

The retinal thickness and choroidal thickness (ChT) were also recorded. Retinal thickness was assessed in the central 1-mm-diameter circle of ETDRS thickness map [central macular thickness (CMT)] and in the treated area using the Spectralis Software (Heidelberg Eye Explorer, Version 1.10.4.0, Heidelberg, Germany). To achieve good choroidal visualization, enhanced depth imaging (EDI) structural OCT was used in all acquisitions. ChT was assessed in the subfoveal area and in the treated area by manually measuring the distance between Bruch's membrane and the sclerochoroidal interface to identify the inner and outer boundaries of the choroid, respectively. To better investigate the treated area, a 49 horizontal raster dense linear B-scans, each composed by 16 averaged OCT B-scans (384 A-scans per line) at $30 \mu \mathrm{m}$ intervals, covering an area of 15 degrees by 5 degrees was performed in each patient at the baseline, at 1-month and 3-month follow-up. All the sections were analyzed in the same place of the retina during the follow-up examinations. In detail, we have obtained the sections in the same place using the follow-up function available on the Spectralis Software (version: 1.10.4.0) ${ }^{25}$.

All the evaluations were also performed in another area of $1.27 \mathrm{~mm}^{2}$ with a similar distance from the fovea that did not receive treatment. This area was used as a control area (i.e. area without treatment). 
Microperimetry. Microperimetry was used to assess changes of retinal sensibility in the customized treated area. After training, all subjects underwent scotopic microperimetry examinations of the central retina in the study eye. Prior to testing, pupil dilatation and dark adaptation were performed. The eyes were fully covered with an opaque eye patch, followed by a waiting period of $30 \mathrm{~min}$ in a dark room $(<0.1 \mathrm{~lx})$. During the first examination, all patients underwent a fast test only for learning. The anatomical position of the fovea was determined by uploading the combined central infrared reflectance image and horizontal B-scan SD-OCT scan of the Spectralis to the MP-1S software. Using the optic nerve head and the major retinal vessels as landmarks for registration to the fundus real-time image of the MP-1S, test stimuli were placed around the fovea (Goldmann size V, $200 \mathrm{ms,}$ 4-2 strategy, background luminance $0.0032 \mathrm{~cd} / \mathrm{m}^{2}$, grid centered on the anatomical position of the fovea). Due to the testing under scotopic conditions, the fixation ring was not necessarily centered on the fovea. The following parameters were recorded in each timepoint: overall retinal sensitivity (MS) of the macular area and the MS of the treated area, fixation percentage calculated within the central $2^{\circ}$ and $4^{\circ 26}$.

Clinical outcome measures. The primary outcome of the PASCAL trial was the safety of treatment measured as retinal sensitivity changes in the treated area 3 months after subthreshold laser treatment (i.e. change in retinal sensitivity).

Prespecified secondary outcomes included:

- Changes in the outer retinal morphology in the treated area using structural OCT during the follow-up;

- Change in mean BCVA during the follow-up;

- Change in the treated area using FAF during the follow-up;

- Adverse and Serious Adverse Events during the follow-up;

- Change in intraocular pressure during the follow-up.

Statistical analysis. A sample size of 16 eyes has a greater than $80 \%$ power to identify a variation of 1.5 decibels in macular sensitivity between pre and post laser treatment assessments, with an estimated standard deviation of the change outcome of 2.0 and an alpha error of 0.05 . Allowing an additional $20 \%$ of the estimated sample size in order to counter possible withdrawn patients, we estimated that 20 eyes would be required in our series.

All statistical analyses were performed using SPSS Statistics Version 20 (IBM, Armonk, New York, USA). In all patients, BCVA was converted to Logarithm of the Minimum Angle of Resolution (LogMAR) for statistical analysis. Categorical variables were expressed as count and percentage, whereas quantitative variables were expressed as mean \pm standard deviation. The agreement between individual measurements from both readers was performed using the intraclass correlation coefficient (ICC; 95\% CI). The Gaussian distribution of continuous variables was verified with the Kolmogorov-Smirnov test. Comparisons of BCVA, CMT, subfoveal ChT, retinal and choroidal thickness in the treated area, number of RPD, ONL thickness, IOP, overall MS of the macular area and of the treated area, fixation percentage in the $2^{\circ}$ and $4^{\circ}$ between different time-points (baseline, 1-month follow-up and 3-month follow-up) were performed using the repeated measures Analysis of Variance (ANOVA) with Bonferroni post-hoc analysis. The comparison between stages of RPD at the baseline and at the end of the follow-up was performed using the Chi-squared test. In all analyses, $p$ values $<0.05$ were considered statistically significant.

\section{Results \\ Patient demographics and main clinical findings. Twenty eyes of 20 patients (mean age $78.4 \pm 6.8$ years, median 77.5, range 67-89) fulfilled the criteria of our study and were included. All patients were Caucasians, 15 were females and 5 males. The fellow eye of 15 patients was affected by nAMD, whereas the fellow eye of 5 patients was affected by dAMD. During the 3 months of the study protocol, patients were not treated with any intravitreal injections or other treatments for AMD in the fellow eye. \\ At the baseline, BCVA was between 20/25 and 20/32 Snellen equivalent (0.140 \pm 0.09 LogMAR; median 0.1; range, 0-0.4) and did not shown significant changes at 1-month and 3-month follow-up (BCVA $0.135 \pm 0.10$ LogMAR; median 0.1; range 0-0.4 and 0.115 \pm 0.09 LogMAR; median 0.1; range 0-0.3 at 1-month and 3-month follow-up, respectively) $(p=0.232)$. None of the included patients gained or lost more than 15 letters during the follow-up. IOP did not show significant changes during the follow-up $(p=0.267)$ (Table 2). Furthermore, no significant changes were disclosed analyzing CMT and subfoveal ChT during the follow-up. In detail, CMT was $275 \pm 19 \mu \mathrm{m}$ (median 275, range 229-305) at the baseline, $276 \pm 19 \mu \mathrm{m}$ (median 276, range 237-320) at 1-month follow-up and $273 \pm 26 \mu \mathrm{m}$ (median 273, range 209-324) at 3-month follow-up ( $p=0.725)$. Subfoveal ChT at the baseline was $223 \pm 110 \mu \mathrm{m}$ (median 187, range 72-426) and did not show significant changes at 1-month and 3 -month follow-up $(223 \pm 112 \mu \mathrm{m}$; median 188; range 77-425 and $221 \pm 107 \mu \mathrm{m}$; median 184; range $72-433$ at 1 -month and 3-month follow-up, respectively) $(p=0.730)$ (Table 2).}

Functional changes in the treated area. Assessment of retinal sensitivity was performed using microperimetry. No significant changes were disclosed before and after the treatment analyzing the overall MS of the macular area and the MS of the treated area. Of note, the overall MS was $13.99 \pm 4.27 \mathrm{~dB}$ at the baseline and did not significantly change during the follow-up $(p=0.152)$ (Table 2, Fig. 2). Analyzing the mean MS of only the treated area, it was $12.08 \pm 4.65 \mathrm{~dB}$ at the baseline, $11.08 \pm 5.06 \mathrm{~dB}$ at 1 -month follow-up, and $11.45 \pm 5.70 \mathrm{~dB}$ at 3 -month follow-up $(p=0.404)$. Furthermore, the mean MS of only the control area was $12.01 \pm 4.67 \mathrm{~dB}$ at 


\begin{tabular}{|c|c|c|c|c|c|c|c|}
\hline & \multicolumn{2}{|l|}{ Baseline } & \multicolumn{2}{|c|}{ 1-month follow-up } & \multicolumn{3}{|c|}{ 3-month follow-up } \\
\hline & Mean \pm SD & $p$ value ${ }^{*}$ & Mean \pm SD & $p$ value $^{\S}$ & Mean \pm SD & $p$ value ${ }^{\varsigma}$ & $p$ value $^{*}$ \\
\hline BCVA, LogMAR & $0.14 \pm 0.09$ & 0.232 & $0.135 \pm 0.10$ & 1.000 & $0.115 \pm 0.09$ & 0.288 & 0.311 \\
\hline IOP, mmHg & $15.5 \pm 2.9$ & 0.267 & $14.9 \pm 2.4$ & 0.827 & $14.7 \pm 2.4$ & 0.300 & 1.000 \\
\hline CMT, $\mu \mathrm{m}$ & $275 \pm 19$ & 0.725 & $276 \pm 19$ & 1.000 & $273 \pm 26$ & 1.000 & 1.000 \\
\hline Subfoveal ChT, $\mu \mathrm{m}$ & $223 \pm 110$ & 0.730 & $223 \pm 112$ & 1.000 & $221 \pm 107$ & 1.000 & 1.000 \\
\hline Retinal thickness (treated area), $\mu \mathrm{m}$ & $295 \pm 26$ & 0.238 & $294 \pm 24$ & 1.000 & $292 \pm 25$ & 0.258 & 0.847 \\
\hline ChT (treated area), $\mu \mathrm{m}$ & $197 \pm 88$ & 0.450 & $195 \pm 94$ & 1.000 & $201 \pm 88$ & 1.000 & 0.613 \\
\hline ONL thickness (treated area), $\mu \mathrm{m}$ & $59.30 \pm 13.50$ & 0.001 & $64.75 \pm 12.31$ & 0.012 & $67.75 \pm 15.52$ & $<0.001$ & 0.213 \\
\hline ONL thickness (control area), $\mu \mathrm{m}$ & $51.61 \pm 10.91$ & 0.199 & $53.39 \pm 12.29$ & 0.645 & $53.56 \pm 12.31$ & 0.204 & 1.000 \\
\hline MS (macular area), $\mathrm{dB}$ & $13.99 \pm 4.27$ & 0.152 & $13.05 \pm 4.19$ & 0.148 & $13.65 \pm 4.70$ & 1.000 & 1.000 \\
\hline MS (treated area), dB & $12.08 \pm 4.65$ & 0.404 & $11.08 \pm 5.06$ & 0.538 & $11.45 \pm 5.70$ & 1.000 & 1.000 \\
\hline MS (control area), dB & $12.01 \pm 4.67$ & 0.120 & $11.02 \pm 4.45$ & 0.112 & $11.69 \pm 5.21$ & 1.000 & 0.910 \\
\hline Fixation percentage $\left(2^{\circ}\right), \%$ & $42.5 \pm 13.2$ & 0.649 & $39.3 \pm 15.1$ & 1.000 & $40.5 \pm 17.3$ & 1.000 & 1.000 \\
\hline Fixation percentage $\left(4^{\circ}\right), \%$ & $83.2 \pm 12.8$ & 0.342 & $78.3 \pm 15.3$ & 0.550 & $80.2 \pm 11.8$ & 0.969 & 1.000 \\
\hline
\end{tabular}

Table 2. Comparisons of anatomical and functional variables between baseline, 1-month follow-up and 3 month follow-up after the treatment. SD standard deviation, BCVA best-corrected visual acuity, IOP intraocular pressure, CMT central macular thickness, ChT choroidal thickness, ONL outer nuclear layer, $M S$ retinal sensitivity. ${ }^{*}$ Analysis of Variance (ANOVA) for paired samples. ${ }^{\S}$ Comparison with baseline using ANOVA for paired samples with Bonferroni post-hoc analysis. "Comparison with 1-month follow-up using ANOVA for paired samples with Bonferroni post-hoc anaysis.

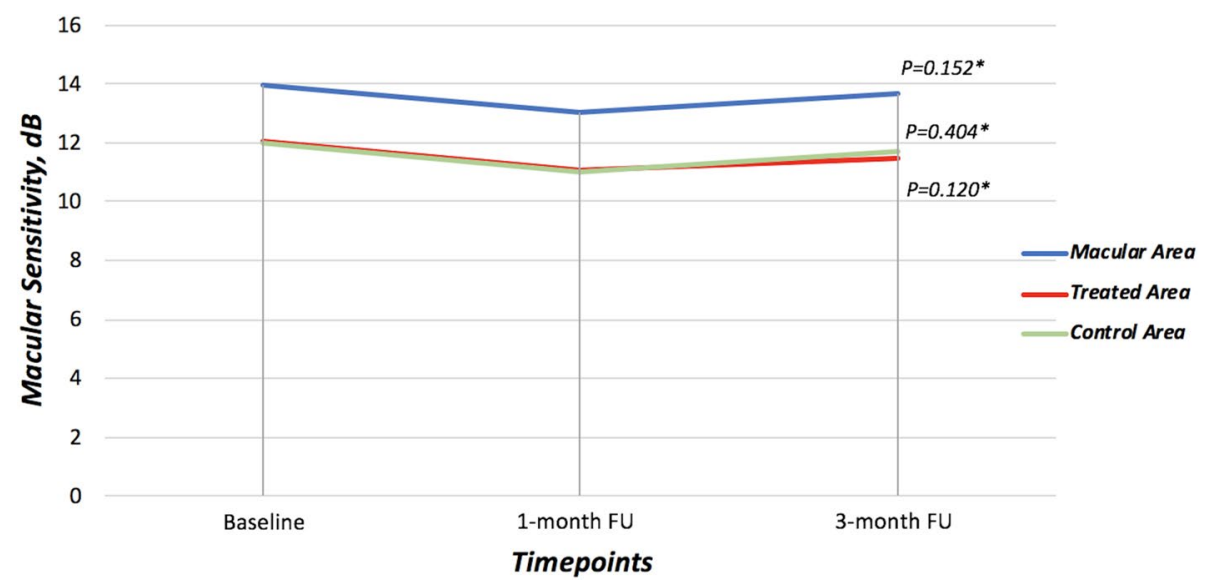

Figure 2. Results of microperimetry in terms of macular sensitivity of the whole macular area, of the treated area, and of the control area during the follow-up. ${ }^{\star}$ Analysis of Variance (ANOVA) for paired samples.

the baseline, $11.02 \pm 4.45 \mathrm{~dB}$ at 1 -month follow-up, and 11.69 \pm 5.21 at 3-month follow-up $(p=0.120)$ (Table 2, Fig. 2).

Mean fixation percentage calculated within the central $2^{\circ}$ (centered on the fovea) was $42.5 \pm 13.2 \%$ at the baseline and did not significantly change during the follow-up $(p=0.649)$; also mean fixation percentage calculated within the central $4^{\circ}$ (centered on the fovea) did not show significant changes during the follow-up $(p=0.342)$ (Table 2).

Anatomical changes in the treated area. An area of $1.27 \mathrm{~mm}^{2}$ was treated in all patients. The area was located at a mean distance of $1904 \pm 544 \mu \mathrm{m}$ (median 1811.5, range 1127-3000) from the fovea. Using structural OCT, we identified a mean of 12.4 $\pm 6.2 \mathrm{RPD}$ (median 11.5, range 3-23) inside the treated area. The distribution of RPD among the RPD stages changed after the treatment $(p<0.001)$. At the baseline, $0.35 \pm 0.59$ RPD were classified as Stage 1, $8.60 \pm 4.79$ as Stage 2, $3.30 \pm 4.35$ as Stage 3, and $0.15 \pm 0.49$ as Stage 4. At 1-month follow-up, $1.20 \pm 1.70 \mathrm{RPD}$ were classified as Stage 1, 8.85 \pm 5.20 as Stage 2, $2.20 \pm 3.98$ as Stage 3, and $0.15 \pm 0.49$ as Stage 4. At 3-month follow-up, 2.30 $\pm 2.18 \mathrm{RPD}$ were classified as Stage 1, $8.75 \pm 4.79$ as Stage 2, $1.15 \pm 2.56$ as Stage 3, and $0.20 \pm 0.70$ as Stage 4 (Fig. 3). This accounted for a significant increase of Stage 1 RPD during the follow-up $(p=0.002)$, no significant changes of Stage 2 RPD $(p=0.909)$, but a significant decrease of Stage 3 RPD $(p=0.020)$ and no significant changes of Stage 4 RPD $(p=0.630)$. This improvement was mainly due to a general improvement in stage 2 and 3 RPD. In detail, $62 \%$ of stage 3 RPD (41 out of 66 stage 3 RPD of all patients) showed an 


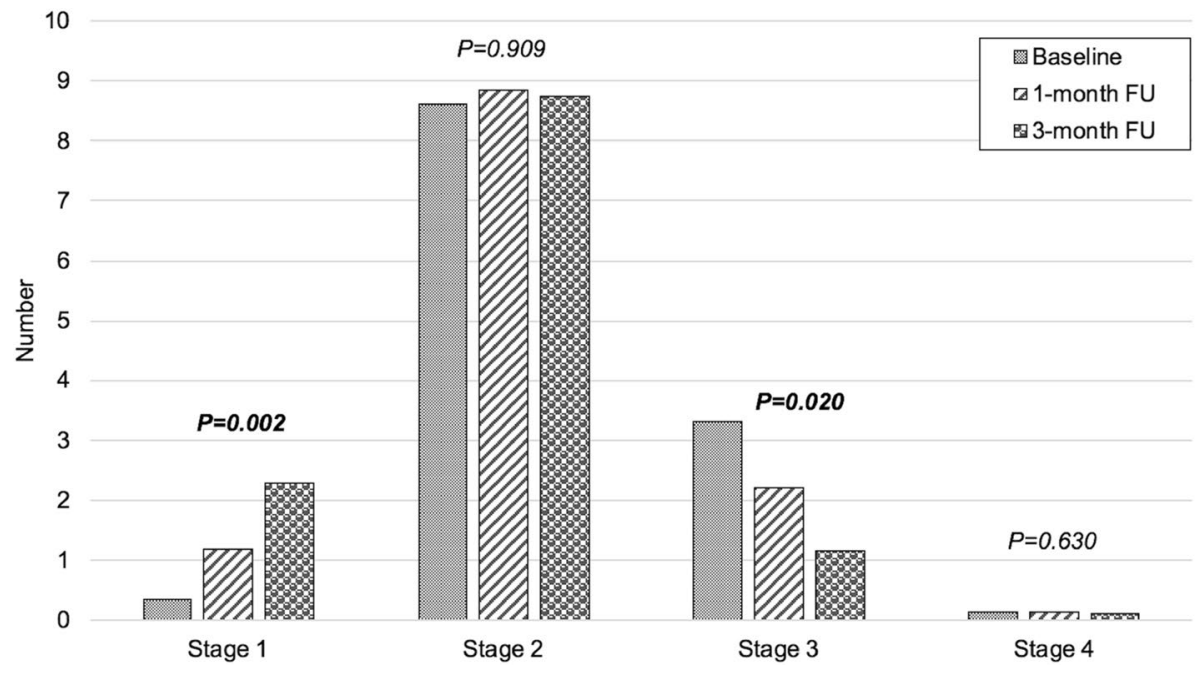

Figure 3. Number of reticular pseudodrusen (RPD) in the treated area according to the stage before and after subthreshold laser treatment. Stage 1 RPD significantly increased after the subthreshold laser treatment (first histogram) due to the significant regression of stage 3 RPD (third histogram). No significant differences were disclosed after the treatment in stage 2 and stage 4 RPD (second and fourth histograms, respectively).

improvement during the 3-month follow-up (28 out of 66 RPD from stage 3 to stage 2, and 13 out of 66 RPD from stage 3 to stage 1). Furthermore, 16\% of stage 2 RPD (27 out of 172 of stage 2 RPD of all patients) showed an improvement to stage 1 during the 3 -month follow-up.

Another area of $1.27 \mathrm{~mm}^{2}$ with a similar distance from the fovea $(1901 \pm 528 \mu \mathrm{m}, p=0.689)$ was selected as a control area (i.e. area without treatment). In the control area, we identified a mean of $9.6 \pm 4.6$ RPD (median 9.5, range 4-20) $(p=0.127)$. Analyzing the distribution of RPD among the RPD stages in the control area (i.e. area without treatment), no significant changes were observed during the follow-up in different stages of RPD. In detail, at the baseline, $0.56 \pm 0.24 \mathrm{RPD}$ were classified as Stage $1(0.50 \pm 1.04$ and $0.22 \pm 0.55 \mathrm{RPD}$ at 1 -month and 3 -month follow-up, respectively; $p=0.157)$. At the baseline, 5.61 $\pm 3.15 \mathrm{RPD}$ were classified as Stage $2(5.67 \pm 3.61$ and 6.06 $\pm 3.56 \mathrm{RPD}$ at 1 -month and 3-month follow-up, respectively; $p=0.305$ ), $3.83 \pm 3.75 \mathrm{RPD}$ were classified as Stage 3 (3.28 \pm 3.89 and 3.22 $\pm 3.73 \mathrm{RPD}$ at 1 -month and 3-month follow-up, respectively; $p=0.251)$, and $0.11 \pm 0.32 \mathrm{RPD}$ were classified as Stage $4(0.17 \pm 0.51$ and $0.11 \pm 0.32 \mathrm{RPD}$ at 1 -month and 3-month follow-up, respectively; $p=0.331$ ).

Analyzing the treated area, the thickness of the ONL significantly increased during the follow-up $(p=0.001)$. In detail, mean ONL thickness at the baseline was $59.30 \pm 13.50 \mu \mathrm{m}$ (median 60.5, range 32-84) and increased to $64.75 \pm 12.31 \mu \mathrm{m}$ (median 69, range 41-82) at 1-month follow-up $(p=0.012)$ and to $67.75 \pm 15.52 \mu \mathrm{m}$ (median 71, range 36-93) at 3-month follow-up $(p<0.001)$ (Table 2, Fig. 4). On the other hand, analyzing the control area, the ONL thickness was $51.61 \pm 10.91 \mu \mathrm{m}$ at the baseline (median 55.5, range 22-69) and it did not show any significant change during the follow-up $(53.39 \pm 12.29 \mu \mathrm{m}$, median 54 , range $23-65[p=0.645]$, and $53.56 \pm 12.31 \mu \mathrm{m}$, median 58 , range $17-66$ [ $p=0.204]$ at 1 -month and 3-month follow-up, respectively).

Interobserver variability between readers was excellent for all measurements (ICC $=0.965[0.912-0.986]$ ).

No significant changes during the follow-up were observed analyzing the mean retinal thickness and choroidal thickness in the treated area ( $p=0.238$ and $p=0.450$, respectively) (Table 2$)$.

Analyzing FAF images, no development/extension of atrophic lesions was observed during the follow-up. Furthermore, no changes in the appearance of RPD were observed.

Safety analysis. No topical and/or systemic side effects were reported from the patients during the 3-month follow-up. None of the patients developed a MNV in the treated eye, and no eye developed atrophic lesion during the follow-up detected using FAF. No other retinal changes in the treated area were disclosed using multimodal imaging modality.

\section{Discussion}

Here, we have reported the results of the first pilot study investigating the safety and short-term efficacy of SLT in patients affected by RPD secondary to dAMD. Overall, the results of our single-center, interventional clinical trial have confirmed the safety of end-point management yellow SLT in the treatment of RPD. Indeed, the MS in both macular and treated areas did not show worsening during the 3-month follow-up using the microperimetry. Furthermore, no ocular or systemic side effects were reported in our patients.

"High-density/low-intensity" SLT was first reported in 2005 in the treatment of diabetic macular edema $(\mathrm{DME})^{27}$. Subthreshold laser does not cause retinal damage and has no known adverse treatment effects ${ }^{21,28,29}$. Indeed, it has been demonstrated that both subthreshold infrared laser and subthreshold yellow laser do not cause clinically visible or invisible scars in the macula ${ }^{29,30}$, and that SLT can be used transfoveally in eyes with 20/20 

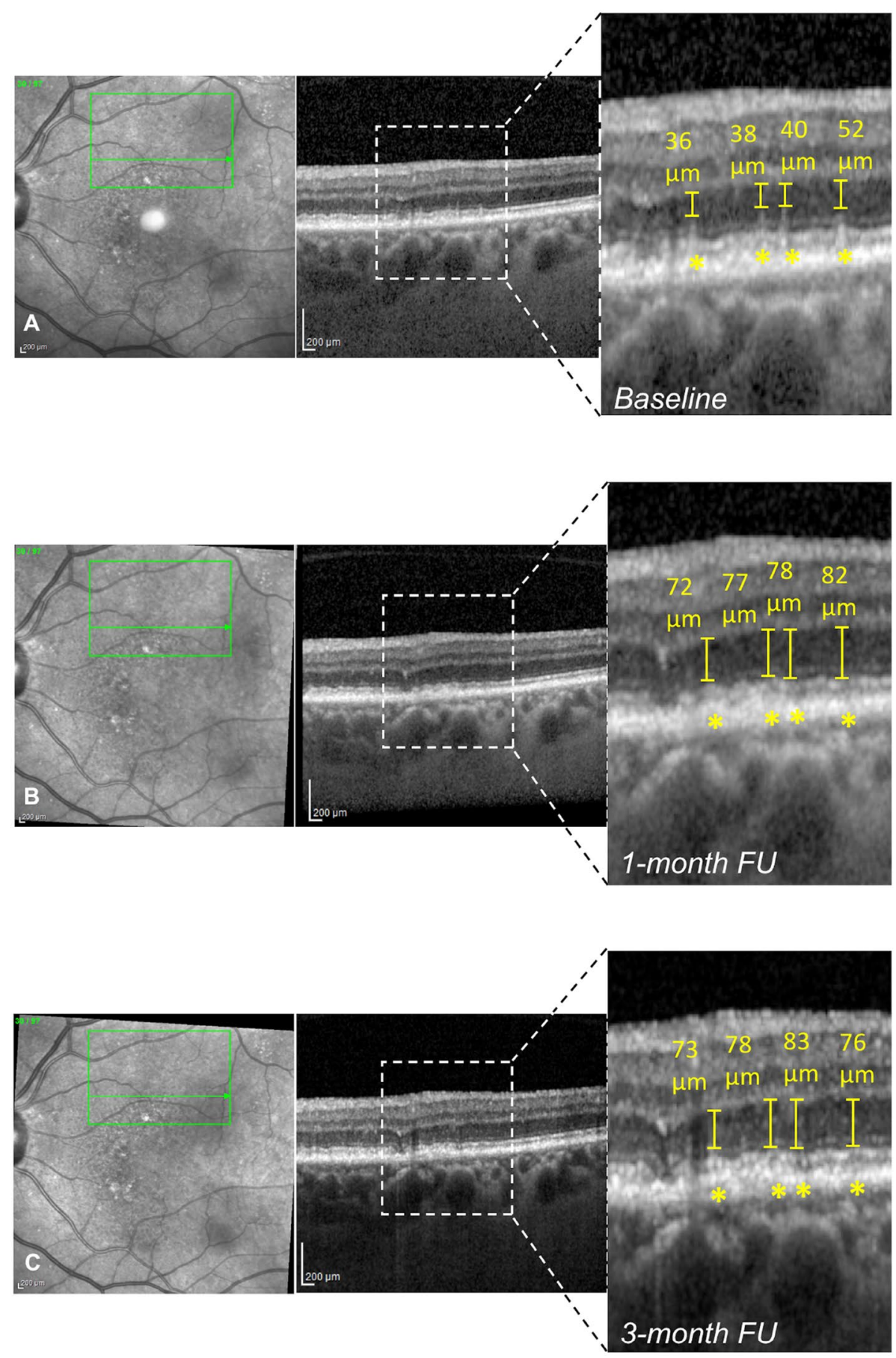

Figure 4. Structural optical coherence tomography (OCT) changes in the morphology of the outer nuclear layer (ONL) before and after subthreshold laser treatment. (A) Combined infrared reflectance (IR) and structural OCT showing the presence of 4 reticular pseudodrusen (RPD) in the treated area at the baseline (magnification). (B, C) Combined IR and structural OCT showing the partial regression of RPD with increased ONL thickness at 1-month (B) and 3-month follow-up (C) (magnifications).

visual acuity to reduce the risk of visual loss caused by early fovea-involving DME ${ }^{24}$. Our data have confirmed that SLT is safe also in patients with high BCVA and dAMD. No local and systemic adverse events were detected after 3 months from the laser treatment (i.e. no slit lamp or fundus abnormal findings, no crystalline lens changes in phakic eyes, no IOP changes, no development of MNV in the treated area).

The PASCAL clinical trial, is a single-center pilot study, evaluating the effect of end-point management yellow SLT in patients with RPD secondary to dAMD. Reticular pseudodrusen deposits are characterized by a widespread disruption and loss of RPE and ellipsoid zone inducing an impaired retinal sensitivity and worse 
visual function already from the early stages of the disease ${ }^{14}$. Furthermore, patients with RPD are characterized by a faster progression to both forms of late $\mathrm{AMD}^{14,18,19}$. It has been suggested that subthreshold laser works by targeting, preserving, and "normalizing" the function of the $\mathrm{RPE}^{31,32}$. Luttrull et $\mathrm{al}^{23}$ have suggested a wider role for subthreshold laser as retinal reparative/protective therapy by re-establishing the function of RPE in patients affected by nAMD, restoring the drug response in drug-tolerant eyes. As subthreshold laser seems to play a role in restoring the function of RPE in patients affected by $\mathrm{AMD}^{23}$ and dysfunction of the RPE has been suggested as the main driving factor in the pathogenesis of RPD, SLT could play a crucial role in the regression of RPD. Our pilot study showed interesting results in the anatomical outcomes of patients treated with subthreshold laser. In detail, analyzing the stages of RPD in the treated area at the baseline and at follow-up examinations, we reported a significant decrease in the number of stage 3 RPD $(p=0.020)$ with, simultaneously, a stage 1 RPD increase at 3 -month follow-up after the laser treatment $(p=0.002)$. Furthermore, according to the down-staging of RPD, we observed a significant increase in the ONL thickness above the treated RPD during the FU $(p=0.001)$. On the other hand, no changes in RPD distribution and no changes in ONL thickness were observed in the control area (i.e. area without treatment). These data suggest a real regression of the RPD rather than a RPD resorption due to the progression of the disease. Indeed, Spaide et $\mathrm{a}^{33}$ reported outer retinal atrophy (i.e. decrease of ONL thickness) in patients with regression of the RPD due to the natural history and evolution of the disease. Contrary, our series demonstrated an increase of the ONL after the reabsorption of RPD secondary to the SLT, supporting the theory of the regression of the disease. These results are of fundamental importance because a regression of the stages of RPD due to SLT could reduce the risk of developing an advanced form of AMD, both neovascular or atrophic. One can argue that our study did not show any significant functional improvement in terms of BCVA or MS in both foveal and treated areas after the treatment. However, we need to account for different aspects. First of all, we treated a small area outside the central fovea, and, for this reason, BCVA may not show any significant change. Secondly, no improvement of MS could be explained by the small treated area and by the short-term follow-up of our clinical trial. On the other side, being a pilot study, a short follow-up was necessary to early detect the possible adverse events. Further studies, with a longer follow-up and a spread treatment involving a larger area, should be performed in order to confirm the results of our pilot study.

Recently, a randomized controlled clinical trial, the LEAD study ${ }^{34}$, demonstrated that subthreshold nanosecond laser (SNL) is a safe treatment but did not significantly reduce the overall rate of the progression to late AMD (both GA and nAMD) in patients with intermediate AMD. However, a post-hoc analysis showed a possible benefit of SNL in patients without RPD and a worse outcome in patients with RPD ${ }^{34,35}$. These data could seem in contrast with our series. However, several differences should be kept in mind. First of all, in the LEAD study, the effect of SNL on patients with RPD was investigated in a post-hoc analysis and not as pre-specified outcome of the study; thus, these data should be taken with caution. Importantly, differently from our study, no detailed morphological and functional analysis of treated RPD was carried out in the LEAD study preventing any definite role of subthreshold laser in the treatment of RPD. Finally, our study is not comparable with the LEAD study because two different kinds of laser were used: the LEAD study used a subthreshold nanopulsed laser, whereas we used a subthreshold continuous laser with end-point management. The different effects on RPD could be due to the different mechanisms of action of the two lasers on the RPE cells.

Despite the encouraging results of the PASCAL clinical trial, it is necessary to clarify the limits of our study, as the low number of patients included in our sample, the short term follow-up, and the margin of error of the retinal Heidelberg tracking system, which may have slightly distorted the lecture of OCT dense scans at the follow-up. However, this is a pilot study aimed to test the safety of the SLT in patients with RPD secondary to $\mathrm{dAMD}$, providing encouraging results and thus suggesting a potential benefit for such approach.

In conclusion, we report the short-term safety of the end-point management yellow SLT with Pascal Synthesis $577 \mathrm{~nm}$ in the treatment of RPD. Furthermore, our results suggest that it may be effective in inducing RPD regression in terms of RPD stage and ONL thickening. However, further clinical studies with larger sample sizes, a sham group, and a longer follow-up are needed in order to confirm these promising results and to test the effects on the progression rate to late AMD of the treated patients.

Received: 23 July 2020; Accepted: 6 January 2021

Published online: 26 January 2021

\section{References}

1. Congdon, N. et al. Causes and prevalence of visual impairment among adults in the United States. Arch. Ophthalmol. 122, 477-485 (2004).

2. Klein, R. et al. Changes in visual acuity in a population over a 15-year period: the Beaver Dam Eye Study. Am. J. Ophthalmol. 142, 539-549 (2006).

3. Rosenfeld, P. J. et al. Ranibizumab for neovascular age-related macular degeneration. N. Engl. J. Med. 355, 1419-1431 (2006).

4. Bandello, F. et al. Recent advances in the management of dry age-related macular degeneration: a review. F1000Research 6, 245 (2017).

5. Sacconi, R., Corbelli, E., Querques, L., Bandello, F. \& Querques, G. A Review of current and future management of geographic atrophy. Ophthalmol. Ther. 6, 69-77 (2017).

6. Biesemeier, A., Taubitz, T., Julien, S., Yoeruek, E. \& Schraermeyer, U. Choriocapillaris breakdown precedes retinal degeneration in age-related macular degeneration. Neurobiol. Aging. https://doi.org/10.1016/j.neurobiolaging.2014.05.003 (2014).

7. Mullins, R. F., Johnson, M. N., Faidley, E. A., Skeie, J. M. \& Huang, J. Choriocapillaris vascular dropout related to density of drusen in human eyes with early age-related macular degeneration. Investig. Ophthalmol. Vis. Sci. https://doi.org/10.1167/iovs.10-6476 (2011).

8. Sacconi, R. et al. Optical coherence tomography angiography in geographic atrophy. Retina 38, 2350-2355 (2018).

9. Sacconi. R. et al. Choriocapillaris flow impairment could predict the enlargement of geographic atrophy lesion. Br. J. Ophthalmol. 105, 97-102 (2021). 
10. Age-Related Eye Disease Study Research Group. Risk factors associated with age-related macular degeneration: a case-control study in the Age-Related Eye Disease Study: AREDS report no3. Ophthalmolgy 107, 2224-2232 (2000).

11. Age-Related Eye Disease Study 2 Research Group. Lutein + zeaxanthin and omega-3 fatty acids for age-related macular degeneration: the Age-Related Eye Disease Study 2 (AREDS2) randomized clinical trial. JAMA 309, 2005-2015 (2013).

12. Meyers, K. J. et al. Joint associations of diet, lifestyle, and genes with age-related macular degeneration. Ophthalmology 122, 2286-2294 (2015).

13. Rabiolo, A. et al. Spotlight on reticular pseudodrusen. Clin. Ophthalmol. 11, 1707-1718 (2017).

14. Querques, G. et al. Impact of reticular pseudodrusen on macular function. Retina 34, 321-329 (2014).

15. Borrelli, E. et al. Impact of bleaching on photoreceptors in different intermediate AMD phenotypes. Transl. Vis. Sci. Technol. 8, 5 (2019).

16. Cicinelli, M. V. et al. Retinal vascular alterations in reticular pseudodrusen with and without outer retinal atrophy assessed by optical coherence tomography angiography. Br. J. Ophthalmol. 102, 1192-1198 (2018).

17. Rabiolo, A. et al. Retinal arterial dilation is impaired in eyes with drusen and reticular pseudodrusen. Retina 39, 2205-2211 (2019).

18. Zweifel, S. A., Imamura, Y., Spaide, T. C., Fujiwara, T. \& Spaide, R. F. Prevalence and significance of subretinal drusenoid deposits (reticular pseudodrusen) in age-related macular degeneration. Ophthalmology 117, 1775-1781 (2010).

19. Querques, G. et al. Pathologic insights from integrated imaging of reticular pseudodrusen in age-related macular degeneration. Retina 31, 518-526 (2011).

20. Vujosevic, S. et al. Subthreshold laser therapy for diabetic macular edema: metabolic and safety issues. Curr. Med. Chem. 20, 3267-3271 (2013).

21. Parodi, M. B. et al. Subthreshold grid laser treatment of macular edema secondary to branch retinal vein occlusion with micropulse infrared (810 nanometer) diode laser. Ophthalmology 113, 2237-2242 (2006).

22. van Dijk, E. H. C. et al. Half-dose photodynamic therapy versus high-density subthreshold micropulse laser treatment in patients with chronic central serous chorioretinopathy: the PLACE trial. Ophthalmology 125, 1547-1555 (2018).

23. Luttrull, J. K., Chang, D. B., Margolis, B. W., Dorin, G. \& Luttrull, D. K. Laser resensitization of medically unresponsive neovascular age-related macular degeneration: efficacy and Implications. Retina 35, 1184-1194 (2015).

24. Querques, G. et al. Analysis of progression of reticular pseudodrusen by spectral domain-optical coherence tomography. Invest. Ophthalmol. Vis. Sci. 53, 1264-1270 (2012).

25. Sacconi, R. et al. Response of central serous chorioretinopathy evaluated by multimodal retinal imaging. Eye (Lond) 32, 734-742 (2018).

26. Steinberg, J. S. et al. Scotopic and photopic microperimetry in patients with reticular drusen and age-related macular degeneration. JAMA Ophthalmol. 133, 690-697 (2015).

27. Luttrull, J. K., Musch, D. C. \& Mainster, M. A. Subthreshold diode micropulse photocoagulation for the treatment of clinically significant diabetic macular oedema. Br. J. Ophthalmol. 89, 74-80 (2005).

28. Chen, S. N., Hwang, J. F., Tseng, L. F. \& Lin, C. J. Subthreshold diode micropulse photocoagulation for the treatment of chronic central serous chorioretinopathy with juxtafoveal leakage. Ophthalmology 115, 2229-2234 (2008).

29. Luttrull, J. K. \& Sinclair, S. H. Safety of transfoveal subthreshold diode micropulse laser for fovea-involving diabetic macular edema in eyes with good visual acuity. Retina 34, 2010-2020 (2014).

30. Vujosevic, S. et al. Subthreshold micropulse yellow laser versus subthreshold micropulse infrared laser in center-involving diabetic macular edema. Retina 35, 1564-1603 (2015).

31. Luttrull, J. K. et al. Long-term safety, high-resolution imaging, and tissue temperature modeling of subvisible diode micropulse photocoagulation for retinovascular macular edema. Retina 32, 375-386 (2012).

32. Luttrull, J. K. \& Dorin, G. Subthreshold diode micropulse laser photocoagulation (SDM) as invisible retinal phototherapy for diabetic macular edema: a review. Curr. Diabetes Rev. 8, 274-284 (2012).

33. Spaide, R. F. Outer retinal atrophy after regression of subretinal drusenoid deposits as a newly recognized form of late age-related macular degeneration. Retina 33, 1800-1808 (2013).

34. Guymer, R. H. et al. Subthreshold nanosecond laser intervention in age-related macular degeneration: the LEAD randomized controlled clinical trial. Ophthalmology 126, 829-838 (2019).

35. Theodore Smith, R. Sub-threshold nanosecond laser (SNL) treatment in intermediate AMD (IAMD). Ann. Eye Sci. 4, 2 (2019).

\section{Author contributions}

G.Q., R.S., F.G., E.B. and F.B.: conception and design of the study; G.Q., R.S., E.B., F.P., I.Z., and L.Q.: patients' enrolment and data acquisition; G.Q. and R.S.: data analysis; All authors: interpretation of data; G.Q. and R.S.: drafting the article; All authors: revising it critically for important intellectual content.

\section{Funding}

Topcon Medical Laser System, Inc. (California, USA) supported the study with a grant.

\section{Competing interests}

Giuseppe Querques is a consultant for: Alimera Sciences (Alpharetta, Georgia, USA), Allergan Inc (Irvine, California, USA), Bayer Shering-Pharma (Berlin, Germany), Heidelberg (Germany), Novartis (Basel, Switzerland), Sandoz (Berlin, Germany), Zeiss (Dublin, USA). Riccardo Sacconi, Francesco Gelormini, Enrico Borrelli, Francesco Prascina, Ilaria Zucchiatti, Lea Querques: none. Francesco Bandello is a consultant for: Alcon (Fort Worth,Texas,USA), Alimera Sciences (Alpharetta, Georgia, USA), Allergan Inc (Irvine, California,USA), Farmila-Thea (Clermont-Ferrand, France), Bayer Shering-Pharma (Berlin, Germany), Bausch And Lomb (Rochester, New York, USA), Genentech (San Francisco, California, USA), Hoffmann-La-Roche (Basel, Switzerland), Novagali Pharma (Évry, France), Novartis (Basel, Switzerland), Sanofi-Aventis (Paris, France), Thrombogenics (Heverlee,Belgium), Zeiss (Dublin, USA).

\section{Additional information}

Supplementary Information The online version contains supplementary material available at https://doi. org/10.1038/s41598-021-81810-7.

Correspondence and requests for materials should be addressed to G.Q.

Reprints and permissions information is available at www.nature.com/reprints. 
Publisher's note Springer Nature remains neutral with regard to jurisdictional claims in published maps and institutional affiliations.

(c) (i) Open Access This article is licensed under a Creative Commons Attribution 4.0 International License, which permits use, sharing, adaptation, distribution and reproduction in any medium or format, as long as you give appropriate credit to the original author(s) and the source, provide a link to the Creative Commons licence, and indicate if changes were made. The images or other third party material in this article are included in the article's Creative Commons licence, unless indicated otherwise in a credit line to the material. If material is not included in the article's Creative Commons licence and your intended use is not permitted by statutory regulation or exceeds the permitted use, you will need to obtain permission directly from the copyright holder. To view a copy of this licence, visit http://creativecommons.org/licenses/by/4.0/.

(C) The Author(s) 2021 